\title{
Left ventricular false tendons are associated with left ventricular dilation and impaired systolic and diastolic function
}

\author{
Michael E. Hall, MD, MS ${ }^{1,2,3}$, Joseph A. Halinski ${ }^{4}$, Thomas A. Skelton, MD1, William F. \\ Campbell, MD ${ }^{1}$, Michael R. McMullan, MD ${ }^{1}$, Robert C. Long, MD, PharmD ${ }^{1}$, Myrna N. \\ Alexander, $\mathbf{M D}^{1}$, James D. Pollard, $\mathbf{M D}^{1}$, John E. Hall, PhD $^{3}$, Ervin R. Fox, MD, MPH${ }^{1}$, \\ Michael D. Winniford, $\mathbf{M D}^{1}$, and Daisuke Kamimura, MD, $\mathbf{P h D}^{1}$ \\ ${ }^{1}$ Division of Cardiology, Department of Medicine, University of Mississippi Medical Center, \\ Jackson, MS \\ ${ }^{2}$ Mississippi Center for Heart Research, University of Mississippi Medical Center, Jackson, MS \\ ${ }^{3}$ Department of Physiology and Biophysics, University of Mississippi Medical Center, Jackson, MS \\ ${ }^{4}$ School of Medicine, University of Mississippi Medical Center, Jackson, MS
}

\section{Summary}

Background-Left ventricular false tendons (LVFTs) are chord-like structures that traverse the LV cavity and are generally considered to be benign. However, they have been associated with arrhythmias, LV hypertrophy and LV dilation in some small studies.

\begin{abstract}
Hypothesis-LVFTs are associated with LV structural and functional changes assessed by echocardiography.
\end{abstract}

\begin{abstract}
Methods-We retrospectively evaluated echocardiographic and clinical parameters of 126 patients identified as having LVFTs within the past 2 years and compared them to 85 age-matched controls without LVFTs.
\end{abstract}

\begin{abstract}
Results-There were no significant differences in age ( $52 \pm 18$ vs $54 \pm 18$ years, $\mathrm{p}=0.37$ ), sex ( 55 vs $59 \%$ men, $\mathrm{p}=0.49$ ), race ( 36 vs $23 \%$ white, $\mathrm{p}=0.07$ ), systolic blood pressure (SBP, $131 \pm 22$ vs $132 \pm 23 \mathrm{mmHg}, \mathrm{p}=0.76$ ) or body mass index (BMI, $31 \pm 8 \mathrm{vs} 29 \pm 10 \mathrm{~kg} / \mathrm{m}^{2}, \mathrm{p}=0.07$ ) between controls and patients with LVFTs, respectively. Patients with LVFTs had more prevalent heart failure ( $43 \%$ vs $21 \%$, $\mathrm{p}=0.001$ ). Patients with LVFTs had more LV dilation, were 2.5 times more likely to have moderate to severe mitral regurgitation, had more severe diastolic dysfunction and reduced LV systolic function (18\% lower) compared with controls (all $\mathrm{p}<0.05$ ). After adjustment for covariates, basal and middle LVFT locations were associated with reduced LV systolic function $(\mathrm{p}<0.01)$ and middle LVFTs were associated with LV dilation $(\mathrm{p}<0.01)$.
\end{abstract}

\footnotetext{
Address correspondence: Michael E. Hall, MD, MS, Division of Cardiology, Department of Medicine, University of Mississippi Medical Center, 2500 North State Street, Jackson, Mississippi, 39216, Tel. 601-984-5678; fax: 601-984-5638; mehall@ umc.edu. Publisher's Disclaimer: This is a PDF file of an unedited manuscript that has been accepted for publication. As a service to our customers we are providing this early version of the manuscript. The manuscript will undergo copyediting, typesetting, and review of the resulting proof before it is published in its final citable form. Please note that during the production process errors may be discovered which could affect the content, and all legal disclaimers that apply to the journal pertain.

None of the authors have any significant conflicts of interest to disclose.
} 
Conclusions-Our findings suggest LVFTs may not be benign variants and basal and middle LVFTs may have more deleterious effects. Further prospective studies should be performed to determine their pathophysiological significance and if they play a causal role in LV dysfunction.

\section{Keywords}

myocardial band; left ventricle; cardiac dysfunction

\section{Introduction}

Left ventricular false tendons (LVFTs) are fibromuscular bands that traverse the left ventricular (LV) cavity and are generally considered to be benign. ${ }^{1}$ Studies of infants demonstrated a high prevalence $(\sim 78 \%)$ of LVFTs on echocardiography and histopathologic analyses of these structures from congenital heart disease patients reveal that most are comprised of fibrous tissue while some are fibromuscular. ${ }^{2}$ They may also contain conduction tissue (Purkinje fibers) and have been identified as a source of ventricular arrhythmias. ${ }^{2,3}$

LVFTs were identified in only 101 out of 1206 echocardiograms from participants in the Framingham Heart Study and these were most commonly located near the apex of the LV. ${ }^{4}$ These investigators found that LVFTs were associated with innocent murmurs, the presence of left ventricular hypertrophy (LVH) as assessed by electrocardiogram (ECG) and were more commonly observed in participants with reduced LV systolic function. However, after multivariable adjustment, only the relationships between LVFTs and ECG-LVH (odds ratio 4.4) and innocent murmurs (odds ratio 5.5) remained significant. Overall, they did not observe an increased risk of mortality in participants with LVFTs. Major limitations of this study include older echocardiographic techniques (studies were performed 1988-1989) and an all-white population. Additionally, participants with LVFTs were more likely to have lower BMIs suggesting that it may be easier to visualize these intracardiac structures in thinner patients.

Other studies have observed varying prevalence of LVFTs and some have noted increased prevalence in patients with dilated ventricles. ${ }^{5}$ It is possible that LVFTs are more commonly noted in patients with dilated LVs as LV dilation may increase the tautness of the bands throughout the cardiac cycle rendering them more visible on echocardiography. ${ }^{5}{ }^{6}$ Some have even hypothesized that LVFTs may prevent LV dilation by acting as a tethering device, particularly in cases of mitral regurgitation. ${ }^{6}$

To date, LVFTs have been associated with abnormal cardiac remodeling in relatively small studies that include almost all-white populations. ${ }^{4,5}$ While there is limited published data on the relationships of LVFTs and LV systolic dysfunction, there is little to no investigation of their association with LV diastolic dysfunction. Therefore, our aim was to evaluate the associations between LVFTs and LV structure and function (both systolic and diastolic) in an ethnically diverse patient population. 


\section{Methods}

\section{Study population}

This retrospective study was approved by the Institutional Review Board (IRB \#2014-0327) at the University of Mississippi Medical Center, a tertiary referral center for the state of Mississippi where over 10,000 echocardiograms are performed yearly. Due to the retrospective study design informed consent was waived and all data was de-identified to protect subjects' privacy. All Over the past 2 years (since 2014), 111 patients were identified by echocardiography as having LVFTs by board certified cardiologists. An LVFT was diagnosed if an echodense band could be visualized traversing the LV cavity in more than one echocardiographic plane. Careful consideration was made not to include mitral valve chordae tendinae or ventricular trabeculations. Then the LV was divided into thirds (apical, middle and basal sections) to describe the location of the LVFTs. A random list of age-and sex-matched controls was generated from patients who had undergone echocardiograms during the same time period as patients identified with LVFTs (Example, Figure 1). From this list, nonconsecutive matched controls were selected under blinding of all clinical (besides age and sex) and echocardiographic data (besides date of echo). Of note, 15 of the matched control patients had LVFTs and were included in the LVFT group (total $n=126$ ). Echocardiograms from both inpatient and outpatient settings were included in the analyses. A blinded reviewer extracted sociodemographic data from patient charts. Clinical data including heart failure diagnoses were abstracted from the patients' medical records and phyisician-listed diagnoses.

\section{Echocardiography}

Echocardiography was performed using an iE33 ultrasound with a multiple frequency transducer (Phillips Medical Systems, Andover, Massachusetts). Standard echocardiographic views were obtained per American Society of Echocardiography (ASE) guidelines. ${ }^{7}$ LV volumes and dimensions were measured from 2D images in the parasternal long axis view per ASE guidelines. ${ }^{7}$ Calculations of $\mathrm{LV}$ mass were made using the following equation as recommended by the American Society of Echocardiography: LV mass index $\left(\mathrm{g} / \mathrm{F}^{\mathrm{m} 2}\right)=$ $\left(0.8^{*}\{1.04 *[(\mathrm{LVDd}+\mathrm{IVSd}+\mathrm{PWd}) 3-(\mathrm{LVDd}) 3]\}+0.6\right) /$ body surface area. LVDd is the left ventricular diastolic dimension, IVSd is the interventricular septum thickness at enddiastole, and PWd is posterior wall thickness at end-diastole. Relative wall thickness (RWT) was calculated as $2 *(\mathrm{PWd}) / \mathrm{LVDd}$. $\mathrm{LV}$ ejection fraction was visually estimated by board certified cardiologists. Transmitral Doppler flow was recorded in the apical 4-chamber view and peak early diastolic (E), peak atrial velocity (A) and E/A ratios were measured. From this same view, septal and lateral mitral annular tissue velocities $\left(e^{\prime}\right)$ were measured using tissue Doppler imaging. The ratios of $\mathrm{E}$ to $\mathrm{e}^{\prime}$ for the septal and lateral mitral annulus were calculated and averaged. Diastolic function grade was assessed and recorded as grade I-III using validated techniques. ${ }^{8}$ Mitral valve regurgitation severity was determined using visual assessment and by measures of vena contracta width and proximal isovelocity surface area regurgitant volumes/fraction where appropriate. ${ }^{9}$ Left atrial diameters were measured in the parasternal long axis view at end-systole and left atrial volumes were assessed by modified Simpson's method from apical 2 chamber view. LVFTs were noted by board certified cardiologists and confirmed by an independent reader. The locations of LVFTs were 
identified as basal, middle or apical LV. Right ventricular systolic function was assessed using tricuspid annular plane systolic excursion (TAPSE). ${ }^{10}$

\section{Statistical Analysis}

All statistical analyses were performed using SAS Enterprise Guide 6.1 (SAS Institute, Cary, North Carolina) and STATA version 14 (STATA Corp, College Station, Texas). Student's ttests were performed to evaluate the differences in mean values between patients with LVFT's and age- and sex-matched controls. The associations between LVFT locations and LV systolic function (LV ejection fraction) and LV dilation (LV end-diastolic dimension) were assessed using multivariable linear regression analyses. A 2 -sided p-value of $<0.05$ was considered statistically significant for all analyses.

\section{Results}

\section{Patient characteristics}

Data from 126 patients with LVFTs and 85 age-and sex-matched patients were analyzed. There were no significant differences in age, sex, race, SBP or BMI between patients with LVFTs and controls (Table 1). Furthermore, the two groups had similar prevalence of hypertension, diabetes and coronary heart disease. Patients with LVFTs had a significantly higher prevalence of heart failure diagnosis $(\mathrm{p}=0.001)$.

\section{LVFTs are associated with LV dilation and impaired LV systolic function}

Compared to control patients, patients with LVFTs had more LV dilation as determined by both LV internal dimensions at end-diastole and end-systole ( $\mathrm{p}<0.001$ and $\mathrm{p}<0.0001$, respectively, Table 2). LV PWd was slightly lower in patients with LVFTs ( $\mathrm{p}=0.049)$ but there were no significant differences in LV IVSd. RWT was significantly lower in LVFT patients $(\mathrm{p}=0.001)$ consistent with $\mathrm{LV}$ dilation and wall thinning. $\mathrm{LV}$ mass indexed to body surface area was significantly higher in LVFT patients $(\mathrm{p}=0.04)$. LV ejection fraction was $18 \%$ lower in patients with LVFTs compared to controls ( $\mathrm{p}<0.001$ ). RV systolic function as assessed by TAPSE was also lower in patients with LVFTs however this was not quite statistically significant $(\mathrm{p}=0.058)$.

\section{LVFT location was associated with LV size and systolic function}

Using multivariable linear regression, LVFT location in either the basal or middle LV myocardial segments was associated with reduced LV ejection fraction ( $<<0.01$ for both) after adjustment for age, sex, race, BMI and systolic blood pressure whereas apical location was not (Table 3). LVFT location at the middle LV was significantly associated with dilated LV ( $\mathrm{p}<0.01$ ), and a basal LVFT location was almost associated with LV dilation ( $\mathrm{p}=0.07)$. And apical LVFT location was not associated with either reduced LV ejection fraction or dilation.

\section{LVFTs are associated with LV diastolic dysfunction}

There were no differences in $\mathrm{E}$ or $\mathrm{A}$ wave velocities or E/A ratios between the two groups. There were also no differences in septal or lateral mitral annular velocities measured by 
tissue Doppler. Mean (septal and lateral) E/e', a surrogate marker for LV filling pressure, was higher in patients with LVFTs ( $\mathrm{p}=0.02$, Figure 2 , Panel A). There were also more LVFT patients with grade II or III diastolic dysfunction compared to controls ( $\mathrm{p}=0.04$, Figure 2, Panel B).

\section{LVFTs are associated with more mitral regurgitation}

Mitral regurgitation severity was classified as mild, moderate, severe or none. Patients with LVFTs were 2.5 times more likely to have moderate or severe mitral regurgitation compared with control patients.

\section{Discussion}

In this study, we evaluated the relationships between LVFTs and measures of LV structure and function using echocardiography in a diverse patient population in a real-world clinical setting. In our study, patients with LVFTs had a higher prevalence of heart failure. We also found that patients with LVFTs had more LV dilation, were more likely to have moderate to severe mitral regurgitation, had more severe LV diastolic dysfunction and significantly reduced LV systolic function compared to age- and sex-matched controls without LVFTs. Furthermore, a basal or middle LVFT location was associated with reduced LV systolic function and LV dilation. To our knowledge, this is the first study to demonstrate an association between LVFTs and reduced LV systolic and diastolic function. Our findings suggest that LVFTs are associated with adverse structural and functional LV changes.

Other studies have demonstrated an association of LVFTs in patients with dilated LVs. ${ }^{5}$ It has been hypothesized that LVFTs are more easily visualized when they are stretched out as occurs when the LV chamber dilates. Some have even hypothesized that LVFTs prevent LV dilation, particularly in the setting of significant mitral regurgitation. ${ }^{6}$ In fact, the Coapsys device, a piece of cord threaded through a patient's LV and tethered with two pads on either side of the heart to mimic the effect of a LVFT, was created to prevent LV dilation in patients with ischemic heart disease and mitral regurgitation. ${ }^{6,8}$ In a randomized control trial of 165 patients undergoing coronary artery bypass grafting and mitral valve replacement, the addition of a Coapsys device reduced LV dilation and mitral regurgitation severity and also showed an overall survival advantage compared to mitral valve replacement alone. ${ }^{11}$

In contrast to the potential beneficial effects of LVFTs in patients with mitral regurgitation, LVFTs have been associated with adverse cardiac remodeling including LVH. 46 Framingham Heart Study investigators observed a greater than four-fold increased risk of LVH on ECG in white participants with LVFTs. Although they observed a three-fold greater risk of reduced LVEF $(<50 \%)$, this finding was not statistically significant $(\mathrm{p}=0.11){ }^{4}$ Importantly, they did not observe an increase in mortality in study participants with LVFTs. Limitations to this study were low prevalence of LVFTs (101/1206 participants) and an allwhite population. The authors noted that the lack of modern echocardiographic equipment may have led to under-recognition of these structures.

We observed significant relationships between the presence of LVFTs and reduced LV systolic and diastolic function as well as mitral regurgitation severity. Our study is cross- 
sectional and we are not able to determine a cause-and-effect relationship. To our knowledge, this is the first study to evaluate the relationship between LVFTs and LV diastolic function.

The potential mechanisms by which LVFTs may cause reduced LV systolic and diastolic function are unclear. However, it is possible that a band of tissue traversing the LV cavity may lead to intracavitary blood flow obstruction, albeit minor. This minor flow obstruction could theoretically lead to increased turbulence, loss of laminar blood flow and kinetic energy and subsequently increased LV wall stress over time (Figure 3). Ultimately, this could lead to LVH or dilation as well as a reduction in LV systolic and diastolic function and secondary mitral regurgitation. It is possible that LVFTs located in the middle and basal LV regions would cause more alterations in LV blood flow. They would not only cause some degree of flow obstruction as the LV fills (diastole) but also during ejection of blood through the LV outflow tract during systole. Support for this possibility comes from our observation that LVFTs located in the basal and middle LV segments were associated with reduced LV systolic function and more LV dilation while these relationships were not significant for apical LVFTs. While these proposed mechanisms are consistent with our observations, they must be considered speculative until further prospective studies with assessment of both LV systolic and diastolic function are conducted to determine if these structures are associated with adverse outcomes or are benign.

Our study has limitations including cross-sectional study design and single-center site. While our study and others have demonstrated an association with LVFTs and adverse cardiac structure and function, other study findings suggest LVFTs are more benign and that they are detected more easily when the LV dilates. ${ }^{5}$ It is important to note that given the cross-sectional design of our study we cannot determine causality for LVFTs on the observed outcomes. Also, given the higher reported prevalence of LVFTs in studies such as Malouf et al. (particularly in children), ${ }^{5}$ it would not be appropriate at this point to say that patients with LVFTs are at higher risk of developing incident heart failure. Our findings using contemporary echocardiographic techniques including assessment of diastolic function suggest that further prospective studies assessing the risk of incident heart failure in patients with LVFTs are warranted. Our study has strengths including an ethnically diverse patient population and we were able to determine that there does not appear to be a race difference in the prevalence of LVFTs. As many cardiologists consider them benign variants, the true prevalence of LVFTs in our institution was not able to be accurately assessed as they are rarely mentioned in formal echocardiographic reports. This is illustrated by the crossover of $15 \%$ of the controls into the LVFT group. While there may be some selection bias in our study due to the fact that patients who undergo echocardiography are more likely to have abnormal findings, we attempted to minimize this bias by randomly selecting an age- and sex-matched control population of patients from our institution who underwent echocardiography during the same time period. Finally, we evaluated prevalent heart failure which was identified by review of physician diagnoses which have potential for misclassification. 


\section{Conclusions}

In a large, tertiary referral center, patients with LVFTs noted on echocardiography had more prevalent heart failure, LV dilation, $18 \%$ lower LV ejection fractions, were more than twice as likely to have at least moderate mitral regurgitation and had more severe LV diastolic dysfunction compared to age- and sex-matched patients without LVFTs. LVFT location near the middle and basal LV segments was associated with greater reductions in LV systolic function and LV dilation. Our cross-sectional study findings suggest that LVFTs may not necessarily be benign and further prospective studies, specifically with outcomes of heart failure, should be performed to evaluate the effects of LVFTs on LV structure and systolic and diastolic function.

\section{Acknowledgments}

The authors would like to thank Connie Watson for administrative support for this project. MH is funded by a grant from the National Institutes of Health, National Institutes of Diabetes and Digestive and Kidney Diseases (NIDDK) grant 1K08DK099415-01A1 the National Institutes of General Medical Sciences (NIGMS) P20GM104357.

\section{References}

1. Kerber RE. Left ventricular false tendons: benign anatomic variant or ominous warning? J Am Soc Echocardiogr. 2009; 22:746-747. [PubMed: 19501330]

2. Philip S, Cherian KM, Wu MH, et al. Left ventricular false tendons: echocardiographic, morphologic, and histopathologic studies and review of the literature. Pediatr Neonatol. 2011; 52:279-286. [PubMed: 22036224]

3. Thakur RK, Klein GJ, Sivaram CA, et al. Anatomic substrate for idiopathic left ventricular tachycardia. Circulation. 1996; 93:497-501. [PubMed: 8565167]

4. Kenchaiah S, Benjamin EJ, Evans JC, et al. Epidemiology of left ventricular false tendons: clinical correlates in the Framingham Heart Study. J Am Soc Echocardiogr. 2009; 22:739-745. [PubMed: 19423290]

5. Malouf J, Gharzuddine W, Kuayli F. A reappraisal of the prevalence and clinical importance of left ventricular false tendons in children and adults. Br Heart J. 1986; 55:587-591. [PubMed: 3718798]

6. Silberger JJ. Epidemiology of left ventricular false tendons: clinical correlates in the Framingham Heart Study. J Am Soc Echocardiogr. 2009; 22:739-745. [PubMed: 19423290]

7. Lang RM, Bierig M, Devereux RB, et al. Recommendations for chamber quantification: a report from the American Society of Echocardiography's Guidelines and Standards Committee and the Chamber Quantification Writing Group, developed in conjunction with the European Association of Echocardiography, a branch of the European Society of Cardiology. J Am Soc Echocardiogr. 2005; 18:1440-1463. [PubMed: 16376782]

8. Redfield MM, Jacobsen SJ, Burnett JC Jr, et al. Burden of systolic and diastolic ventricular dysfunction in the community: appreciating the scope of the heart failure epidemic. JAMA. 2003; 289:194-202. [PubMed: 12517230]

9. Zoghbi WA, Enriquez-Sarano M, Foster E, et al. American Society of Echocardiography. Recommendations for evaluation of the severity of native valvular regurgitation with twodimensional and Doppler echocardiography. J Am Soc Echocardiogr. 2003; 16:777-802. [PubMed: 12835667]

10. Lang RM, Badano LP, Mor-Avi V, et al. Recommendations for cardiac chamber quantification by echocardiography in adults: an update from the American Society of Echocardiography and the European Association of Cardiovascular Imaging. J Am Soc Echocardiogr. 2015; 28:1-39.e14. [PubMed: 25559473]

11. Grossi EA, Patel N, Woo YJ, et al. RESTOR-MV Study Group. Outcomes of the RESTOR-MV Trial (Randomized Evaluation of a Surgical Treatment for Off-Pump Repair of the Mitral Valve). J Am Coll Cardiol. 2010; 56:1984-93. [PubMed: 21126639] 

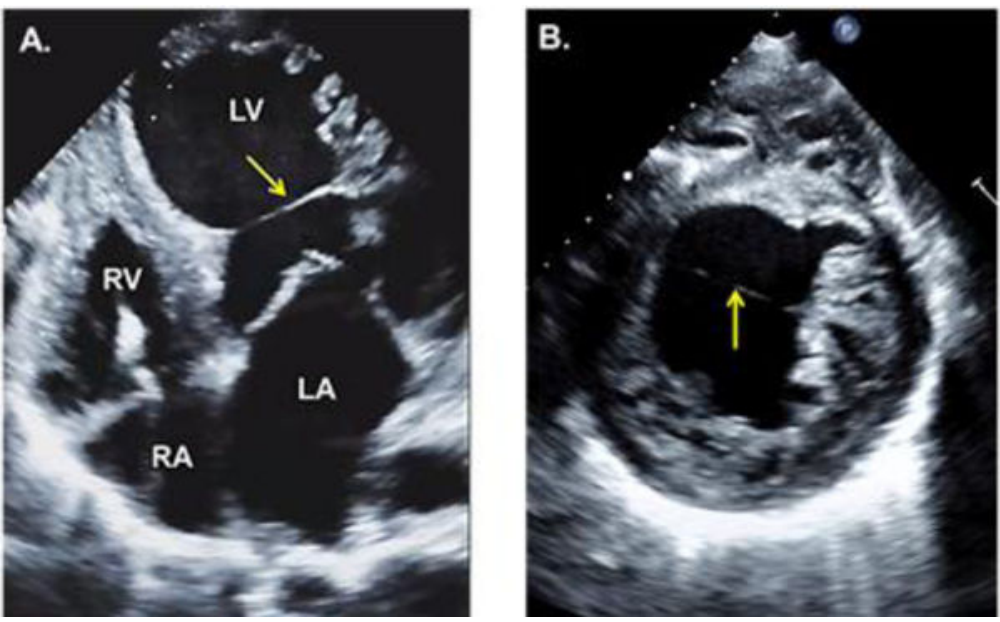

Figure 1.

Panel A. Representative apical four-chamber view with a left ventricular false tendon (yellow arrow) traversing the mid ventricle. Panel B. Mid-ventricular parasternal short axis view from the same patient (false tendon denoted by yellow arrow). 
A.

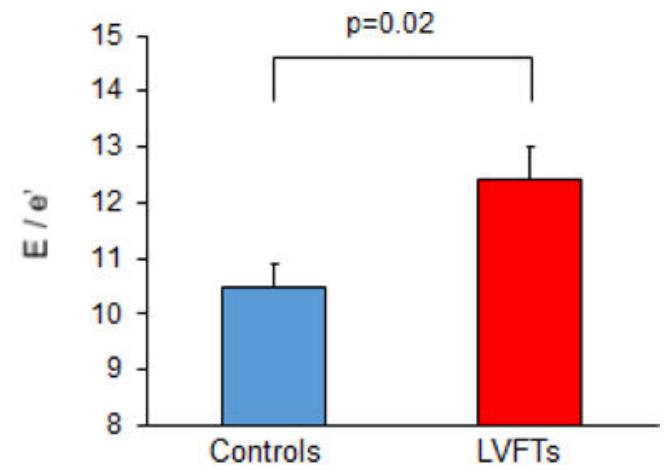

B.

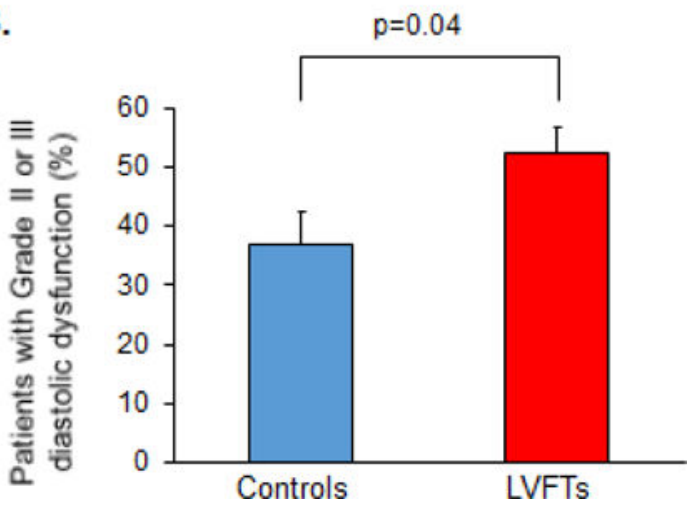

Figure 2.

Panel A. E/e' (a marker of left ventricular end-diastolic pressure) was higher in patients with left ventricular false tendons compared to controls. Panel B. More patients with left ventricular false tendons had evidence of advanced diastolic dysfunction. 
A.

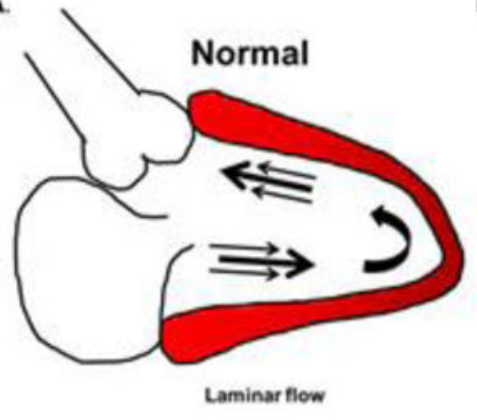

B.

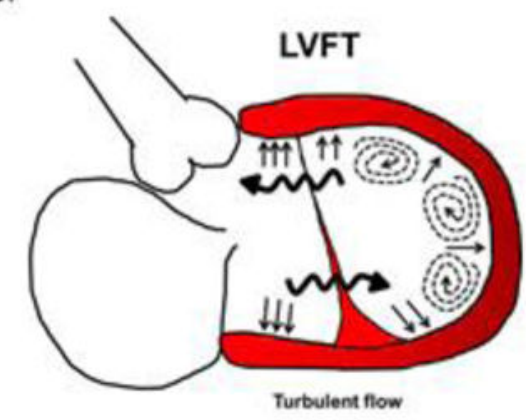

Figure 3.

Proposed mechanism by which left ventricular false tendons may cause increased turbulent flow (Panel B) compared to normal laminar flow (Panel A). Loss of laminar flow may increase left ventricular wall stress and ultimately chamber dilation. 
Table 1

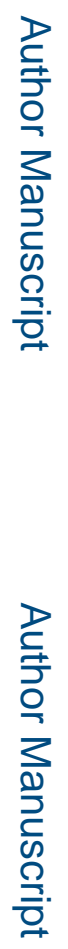

\section{Patient characteristics}

\begin{tabular}{|l|c|c|c|}
\hline & $\begin{array}{c}\text { Controls } \\
(\mathbf{n = 8 5})\end{array}$ & $\begin{array}{c}\text { LVFT } \\
(\mathbf{n = 1 2 6})\end{array}$ & p-value \\
\hline Age (years $\pm \mathrm{SD})$ & $52 \pm 18$ & $54 \pm 18$ & 0.37 \\
\hline Female sex, $\mathrm{n}(\%)$ & $38(45)$ & $51(40)$ & 0.49 \\
\hline African American, $\mathrm{n}(\%)$ & $53(64)$ & $97(77)$ & 0.07 \\
\hline Body Mass Index $\left(\mathrm{kg} / \mathrm{m}^{2} \pm \mathrm{SD}\right)$ & $31 \pm 8$ & $29 \pm 10$ & 0.07 \\
\hline Systolic Blood Pressure $(\mathrm{mmHg} \pm \mathrm{SD})$ & $131 \pm 22$ & $132 \pm 23$ & 0.76 \\
\hline Hypertension diagnosis, $\mathrm{n}(\%)$ & $56(68)$ & $91(76)$ & 0.20 \\
\hline Diabetes diagnosis, $\mathrm{n}(\%)$ & $27(33)$ & $35(30$ & 0.62 \\
\hline Coronary heart disease diagnosis, $\mathrm{n}(\%)$ & $24(29)$ & $28(23)$ & 0.31 \\
\hline History of myocardial infarction, $\mathrm{n}(\%)$ & $9(11)$ & $9(7)$ & 0.39 \\
\hline History of heart failure, $\mathrm{n}(\%)$ & $17(21)$ & $51(43)$ & $0.001 *$ \\
\hline
\end{tabular}


Table 2

Echocardiographic parameters of patients with left ventricular false tendons compared with age-matched controls.

\begin{tabular}{|c|c|c|c|}
\hline & $\begin{array}{c}\text { Controls } \\
(\text { mean } \pm \text { SD })\end{array}$ & $\begin{array}{c}\text { LVFT } \\
(\text { mean } \pm \text { SD) }\end{array}$ & p-value \\
\hline LV posterior wall thickness $(\mathrm{cm})$ & $1.22 \pm 0.28$ & $1.14 \pm 0.28$ & $0.04 *$ \\
\hline LV interventricular septal thickness $(\mathrm{cm})$ & $1.17 \pm 0.27$ & $1.13 \pm 0.31$ & 0.34 \\
\hline LV relative wall thickness $(\mathrm{cm})$ & $0.55 \pm 0.17$ & $0.47 \pm 0.15$ & $0.001^{*}$ \\
\hline LV mass/body surface area $\left(\mathrm{g} / \mathrm{m}^{2}\right)$ & $102 \pm 33$ & $113 \pm 43$ & $0.04 *$ \\
\hline LV internal diameter, end-diastole $(\mathrm{cm})$ & $4.59 \pm 0.68$ & $4.99 \pm 0.93$ & $<0.001^{*}$ \\
\hline LV internal diameter, end-systole $(\mathrm{cm})$ & $3.21 \pm 0.80$ & $3.78 \pm 1.22$ & $<0.001^{*}$ \\
\hline LV ejection fraction $(\%)$ & $55 \pm 13$ & $45 \pm 17$ & $<0.001^{*}$ \\
\hline Mitral E-wave velocity $(\mathrm{cm} / \mathrm{s})$ & $84 \pm 28$ & $88 \pm 25$ & 0.34 \\
\hline Mitral A-wave velocity $(\mathrm{cm} / \mathrm{s})$ & $75 \pm 23$ & $76 \pm 28$ & 0.76 \\
\hline Mitral E/A & $1.2 \pm 0.5$ & $1.3 \pm 0.8$ & 0.19 \\
\hline Mitral septal $\mathrm{e}^{\prime}$ velocity $(\mathrm{cm} / \mathrm{s})$ & $7.2 \pm 2.2$ & $7.1 \pm 2.9$ & 0.69 \\
\hline Mitral lateral $\mathrm{e}^{\prime}$ velocity $(\mathrm{cm} / \mathrm{s})$ & $9.8 \pm 3.4$ & $9.0 \pm 3.5$ & 0.15 \\
\hline E/e' (combined septal and lateral) & $10.5 \pm 3.9$ & $12.4 \pm 6.6$ & $0.02 *$ \\
\hline Left atrial volume index $\left(\mathrm{cm}^{3} / \mathrm{m}^{2}\right)$ & $26.3 \pm 14$ & $29.0 \pm 14$ & 0.21 \\
\hline TAPSE $(\mathrm{mm})$ & $21.4 \pm 6.4$ & $19.7 \pm 5.7$ & 0.05 \\
\hline RV systolic pressure $(\mathrm{mmHg})$ & $35.2 \pm 11.1$ & $38.4 \pm 13.5$ & 0.14 \\
\hline Mitral regurgitation ( $\%$ with moderate to severe regurgitation) & $11 \pm 31$ & $28 \pm 45$ & $0.003 *$ \\
\hline
\end{tabular}

$\mathrm{LV}=$ left ventricle, TAPSE=tricuspid annular plane systolic excursion, $\mathrm{RV}=$ right ventricle 


\section{Table 3}

Multivariable linear regression analyses evaluating the associations of LVFT locations and LV systolic function (LV ejection fraction) and LV dilation (left ventricular internal diameter at end-diastole).

\begin{tabular}{|l|c|c|}
\hline Variable & Coefficient $(\mathbf{9 5 \%} \mathbf{C I})$ & p-value \\
\hline LV systolic function (LVEF) & & \\
\hline Age (years) & $-0.19(-0.31 \sim-0.06)$ & $0.004^{*}$ \\
\hline Sex (male) & $1.45(-2.98 \sim 5.88)$ & 0.52 \\
\hline Race (white) & $-4.72(-9.54 \sim 0.10)$ & 0.06 \\
\hline Body Mass Index (kg/m2) & $-0.11(-0.34 \sim 0.12)$ & 0.36 \\
\hline Systolic Blood Pressure (mmHg) & $0.15(0.05 \sim 0.25)$ & $0.005^{*}$ \\
\hline LVFT Location & & \\
\hline Basal LV & $-7.95(-13.8 \sim-2.1)$ & $0.008^{*}$ \\
\hline Middle LV & $-9.25(-14.4 \sim-4.1)$ & $<0.001^{*}$ \\
\hline Apical LV & $-2.79(-11.9 \sim-6.3)$ & 0.55 \\
\hline LV dilation $(\mathbf{L V I D d )}$ & & \\
\hline Age (years) & $-0.002(-0.01 \sim 0.00)$ & 0.56 \\
\hline Sex (male) & $-0.27(-0.52 \sim-0.02)$ & $0.03^{*}$ \\
\hline Race (white) & $0.08(-0.19 \sim 0.35)$ & 0.56 \\
\hline Body Mass Index (kg/m2) & $0.02(0.00 \sim 0.03)$ & $0.02^{*}$ \\
\hline Systolic Blood Pressure $(\mathrm{mmHg})$ & $-0.005(-0.10 \sim 0.00)$ & 0.10 \\
\hline LVFT Location & & \\
\hline Basal LV & $0.29(-0.03 \sim 0.61)$ & 0.08 \\
\hline Middle LV & $0.39(0.11 \sim 0.68)$ & $0.007^{*}$ \\
\hline Apical LV & $0.17(-0.34 \sim-0.67)$ & 0.52 \\
\hline
\end{tabular}

$L V=$ left ventricle, $L V E F=$ left ventricular ejection fraction, $L V I D d=l e f t$ ventricular internal diameter at end diastole 